\title{
Undersampled Hyperspectral Image Reconstruction Based on Surfacelet Transform
}

\author{
Lei Liu, ${ }^{1}$ Jingwen Yan, $^{2}$ Di Guo, ${ }^{3}$ Yunsong Liu, ${ }^{4}$ and Xiaobo Qu ${ }^{4}$ \\ ${ }^{1}$ Department of Mathematics, Shantou University, Shantou 515063, China \\ ${ }^{2}$ Guangdong Provincial Key Laboratory of Digital Signal and Image Processing, Shantou University, Shantou 515063, China \\ ${ }^{3}$ School of Computer and Information Engineering, Xiamen University of Technology, Xiamen 361005, China \\ ${ }^{4}$ Department of Electronic Science, Xiamen University, Xiamen 361005, China \\ Correspondence should be addressed to Xiaobo Qu; quxiaobo@xmu.edu.cn
}

Received 7 July 2014; Revised 23 September 2014; Accepted 24 September 2014

Academic Editor: Yongqiang Zhao

Copyright ( 2015 Lei Liu et al. This is an open access article distributed under the Creative Commons Attribution License, which permits unrestricted use, distribution, and reproduction in any medium, provided the original work is properly cited.

\begin{abstract}
Hyperspectral imaging is a crucial technique for military and environmental monitoring. However, limited equipment hardware resources severely affect the transmission and storage of a huge amount of data for hyperspectral images. This limitation has the potentials to be solved by compressive sensing (CS), which allows reconstructing images from undersampled measurements with low error. Sparsity and incoherence are two essential requirements for CS. In this paper, we introduce surfacelet, a directional multiresolution transform for 3D data, to sparsify the hyperspectral images. Besides, a Gram-Schmidt orthogonalization is used in CS random encoding matrix, two-dimensional and three-dimensional orthogonal CS random encoding matrixes and a patchbased CS encoding scheme are designed. The proposed surfacelet-based hyperspectral images reconstruction problem is solved by a fast iterative shrinkage-thresholding algorithm. Experiments demonstrate that reconstruction of spectral lines and spatial images is significantly improved using the proposed method than using conventional three-dimensional wavelets, and growing randomness of encoding matrix can further improve the quality of hyperspectral data. Patch-based CS encoding strategy can be used to deal with large data because data in different patches can be independently sampled.
\end{abstract}

\section{Introduction}

Typical hyperspectral imaging (HSI) is acquired on satellites and aerospace probes and then transmitted to grounds. The imaging spectrometer can provide tens to hundreds of narrow-band spectral information for each spatial location. The huge amounts of data but scarce equipment hardware resources on satellites and aerospace severely limit the transmission and storage of hyperspectral images $[1,2]$. However, the ground receiving side holds very strong processing capability. If we transfer the system complexity from the satellites and aerospace probes to the ground, it is expected to potentially solve the limitation in traditional high spectral sampling.

Traditionally, super-resolution reconstruction is used to improve the spatial resolution of HSI $[3,4]$ while compression can improve the transmission efficiency [5-8]. We acquire "all" data and then "throw away" most of it in transmission process. Can we just directly measure the part that we need to save the cost of storage? Compressed sensing (CS) has been proposed to solve these contradictions in remote sensing. The theory of CS shows that a sparse signal can be recovered from a relatively small number of linear measurements $[9,10]$. The difference between the conventional method and CS based transmission method is shown in Figure 1.

CS has been widely used in medical imaging $[11,12]$ and wireless sensor network $[13,14]$ to reduce the sampled data in recent years which has been applied in remote sensing. Duarte et al. [15] proposed a single-pixel imaging method. Ma [2] applied CS in single frame imaging. Aravind et al. [16] used ten spectral bands to compare orthogonal matching pursuit with simultaneous orthogonal matching pursuit in reconstruction. Fowler [17] presented compressiveprojection PCA to effectively shift the computational burden of PCA from the encoder to the decoder. 


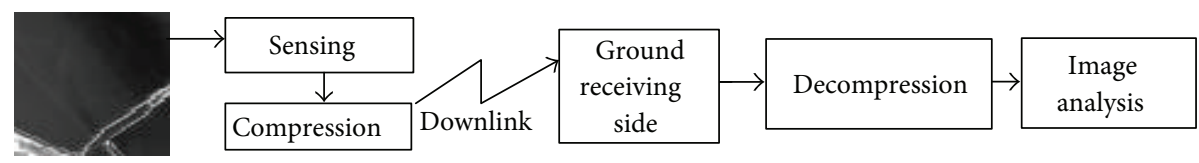

(a)

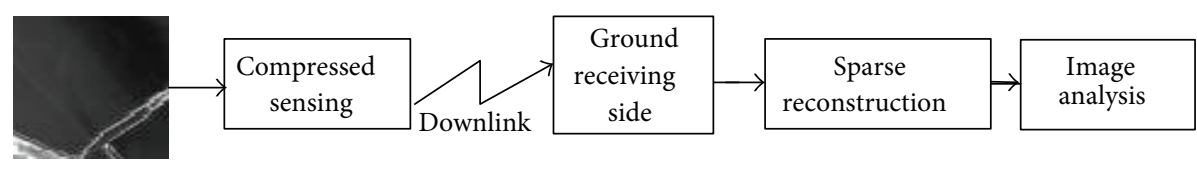

(b)

FIgURE 1: Conventional HSI transmission and CS HSI transmission. (a) Conventional sampling, compression, and decompression. (b) Compressed sensing and sparse reconstruction.

According to the CS theory, the reconstruction error is bounded by the sparse approximation error [18]. For a signal $\mathbf{x} \in \mathbb{R}^{N}$, let $\widetilde{\mathbf{x}}$ be the solution to obey CS conditions, $\Psi^{T}$ a forward transform, and $\left(\Psi^{T} \mathbf{x}\right)_{s}$ the sparse approximation of $\mathbf{x}$ in transform domain by retaining only the $s$-largest entries of $\Psi^{T} \mathbf{x}$; then, the reconstruction error is

$$
\|\widetilde{\mathbf{x}}-\mathbf{x}\|_{2} \leq C_{0} \varepsilon+\frac{C_{1}}{\sqrt{S}}\left\|\Psi^{T} \mathbf{x}-\left(\Psi^{T} \mathbf{x}\right)_{s}\right\|_{1},
$$

where $C_{0}, C_{1}$ are small positive constants and $\varepsilon$ is noise power [19]. Equation (1) implies that a sparser representation will reduce the reconstruction error. An optimal sparsifying transform is always important for sparse image reconstruction to reduce the reconstruction error.

In this study, we introduce the surfacelet transform (ST) to sparsely represent hyperspectral images by making use of the spatial and spectral information. The advantage of ST over wavelet on sparse HSI data reconstruction is demonstrated, where the data are reconstructed by using a fast iterative shrinkage-thresholding algorithm [20]. To further improve the reconstruction performance, a 3-dimensional (3D) random encoding is designed and the Gram-Schmidt orthogonalization is adopted. Finally, a patch-based CS encoding scheme is designed to deal with large size data.

The remainder of this paper is organized as follows. First, ST-based compressive sensing HSI reconstruction method is introduced in Section 2. The experimental results are presented in Section 3. Finally, conclusions are given in Section 4 .

\section{ST-Based Compressive Sensing HSI Reconstruction}

The CS theory comes up with two principles: sparsity, which asserts that the reconstructed signal is sparse with a transform $\Psi$, and incoherence, which requires that the encoding matrix $\Phi$ is incoherent with $\Psi[10]$.

\subsection{Sparse Representation of HSI Using Surfacelet Transform.} Wavelet is commonly used as a typical sparse transform for HSI [21-24]. However, wavelet sometimes fails in sparsely representing HSI because it has only 7 directions [25-27].
Surfacelet transform (ST), proposed by Lu and Do [25], can efficiently capture the surface intrinsic geometrical structure within $N$-dimensional signals [25]. It offers $3 \times 2^{L}$ directional subbands with decomposition level $L$ by combining the multiscale pyramid with the 3-dimensional directional filter banks (3D-DFB). Thus, surfacelet with more directions may help reducing the blocky artifacts caused by orthogonal wavelets $[24,25]$.

ST is a multiscale version of the $3 \mathrm{D}-\mathrm{DFB}$. The input signal $x[n]$ first goes through the $3 \mathrm{D}$ hourglass filter $P_{0,0}^{0,0}(\omega)$, which is a three-channel undecimated filter bank. One branch of the three-channel structure of 3D-DFB is given by Figure 2 . The output $y[n]$ is then fed into a $2 \mathrm{D}$ filter bank, denoted by $\operatorname{IRC}_{12}^{\left(l_{2}\right)}$, which operates on the $\left(n_{1}, n_{2}\right)$ planes. The treestructured filter bank $\operatorname{IRC}_{12}^{\left(l_{2}\right)}$ produces $2^{l_{2}}$ output subbands, denoted by $z_{i}[n]$ for $0 \leq i \leq 2^{l_{2}}$. Each output is then fed into another $2 \mathrm{D}$ filter bank $\operatorname{IRC}_{13}^{\left(l_{3}\right)}$ operating on the $\left(n_{1}, n_{3}\right)$ planes. In the end, we get $2^{l_{2}+l_{3}}$ outputs, represented by $z_{i, j}[n]$ for $0 \leq i \leq 2^{l_{2}}$ and $0 \leq j \leq 2^{l_{3}}$ [25]. $3 \times 2^{L}$ spatial domain basis images of ST with 2 levels of decomposition are shown in Figure 3.

ST is optimal for the $C^{2}$ singularities, thus providing sparser representation of smooth curves and surface singularities than wavelet. Theoretically, for the $C^{2}$ singularities of an image $\mathbf{x}$, the best $S$-term approximation error $\left\|\widetilde{\mathbf{x}}_{s}-\mathbf{x}\right\|_{2}$ using ST has error decay rate of $O\left(S^{-2}\right)$, while this error decay rate is $O\left(S^{-1}\right)$ for typical wavelets $[28,29]$. Figure 4 shows that a sparser representation is achieved using ST. According to the CS theory [19], the reconstruction error bound is proportional to $\left\|\Psi^{T} \mathbf{x}-\left(\Psi^{T} \mathbf{x}\right)_{S}\right\|_{1}$, where $\Psi^{T}$ is the forward transform and $S$ means the number of preserved coefficients in transform domain. Therefore, ST is expected to reduce the image reconstruction error of HSI.

2.2. Incoherence and Gaussian Random Encoding Matrix. Incoherence means a column of the CS encoding matrix must be trying the proliferation in the corresponding sparsity basis $\Psi$. Since this paper focuses on investigating the spatialspectral sparsity of HSI, a Gaussian random matrix is chosen as $\Phi$ because it is incoherent with the entire existing basis 


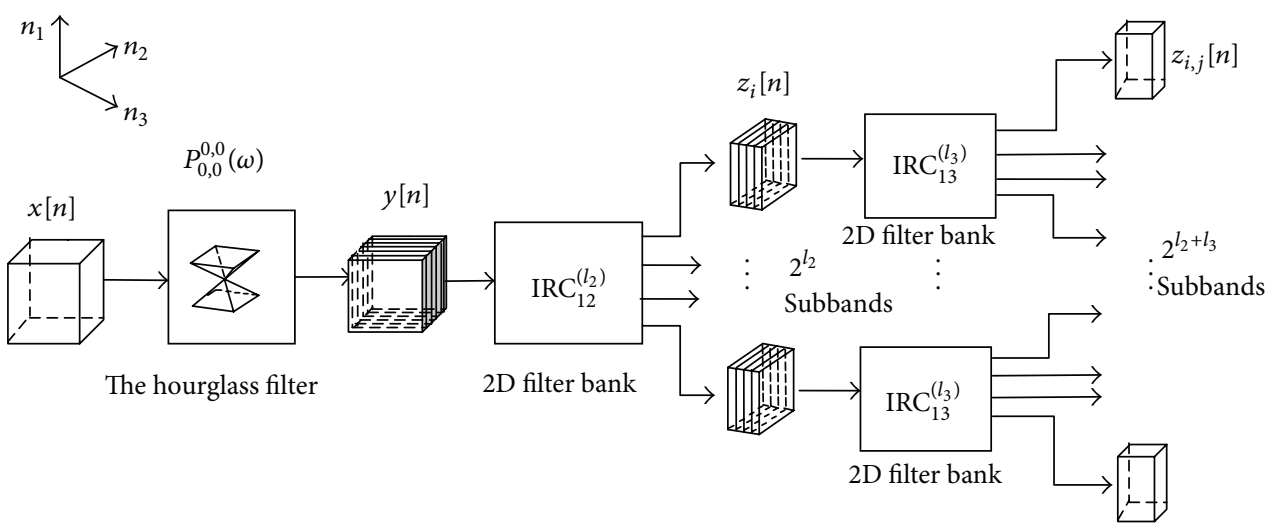

FIGURE 2: One branch of the three-channel structure of 3D-DFB.

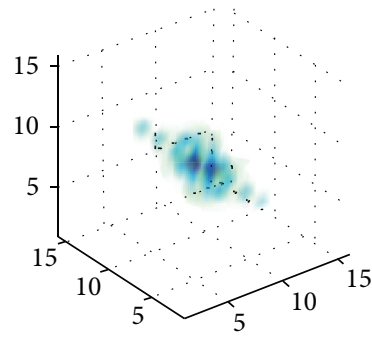

(a)

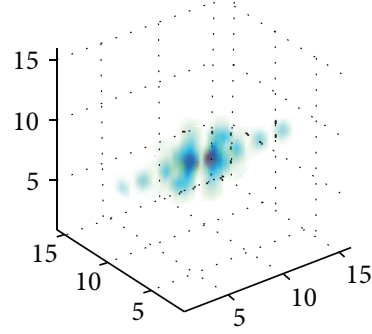

(e)

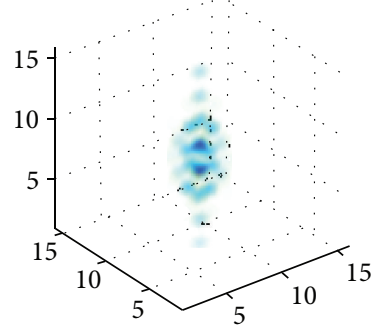

(i)

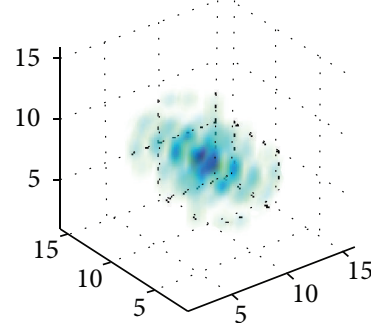

(b)

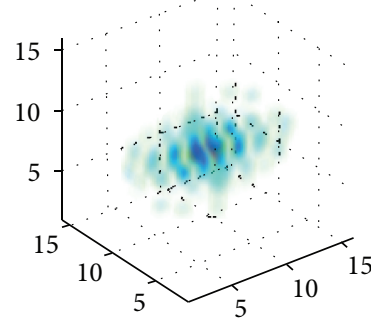

(f)

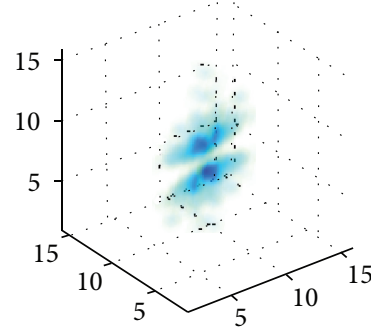

(j)

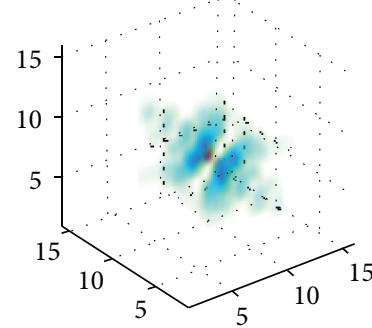

(c)

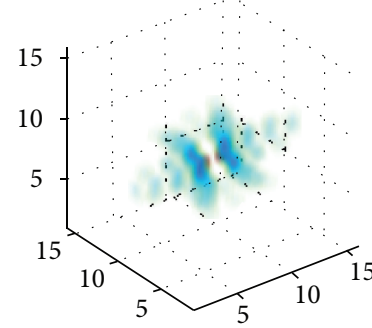

(g)

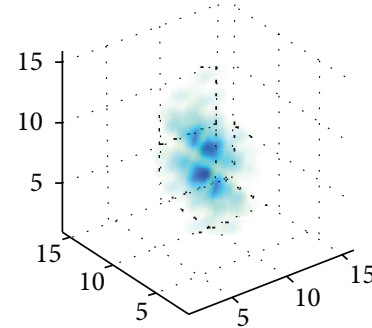

$(\mathrm{k})$

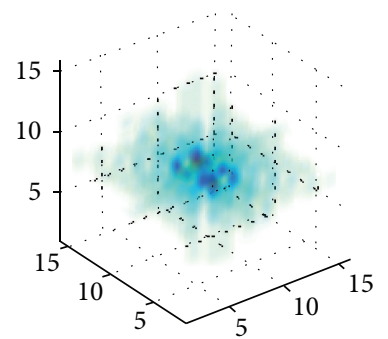

(d)

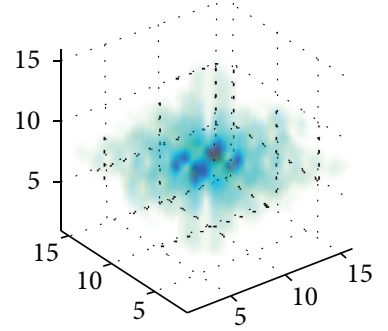

(h)

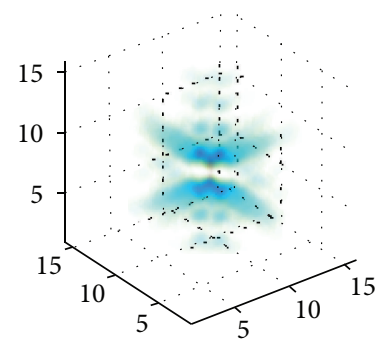

(l)

FIGURE 3: $3 \times 2^{L}$ basis images of ST in 3D spatial domains with 2-level decomposition.

$[9,10]$. If each spatial image is encoded separately, $\boldsymbol{\Phi}$ can be represented by block matrix:

$$
\boldsymbol{\Phi}=\left[\begin{array}{cccc}
\boldsymbol{\Phi}_{1} & \mathbf{0} & \cdots & \mathbf{0} \\
\mathbf{0} & \boldsymbol{\Phi}_{2} & \cdots & \mathbf{0} \\
\vdots & & \ddots & \vdots \\
\mathbf{0} & \mathbf{0} & \cdots & \boldsymbol{\Phi}_{L}
\end{array}\right],
$$

where $\boldsymbol{\Phi}_{i}(1 \leq i \leq L)$ is an encoding matrix for the band $i$ of HSI. When $\Phi_{1}=\Phi_{2}=\cdots=\Phi_{L}$, that is, each 2D image is encoded with the same encoding matrix, then $\Phi$ is called 2D random encoding matrix; when $\boldsymbol{\Phi}_{1} \neq \boldsymbol{\Phi}_{2} \neq \cdots \neq$ $\Phi_{L}$, that is, each 2D image is encoded differently, then $\Phi$ is called 3D random encoding matrix. Physically, 3D random encoding means that digital micromirror device (DMD) 


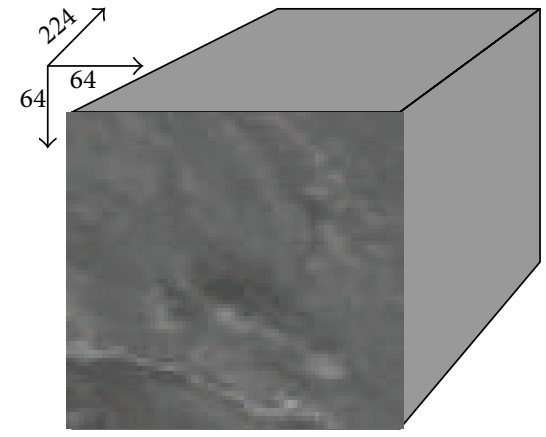

(a)

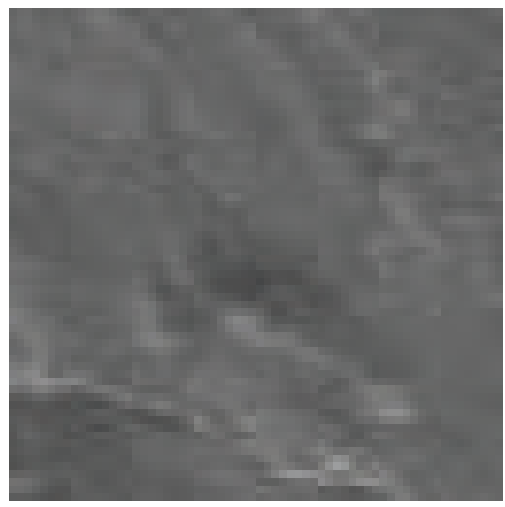

(c)

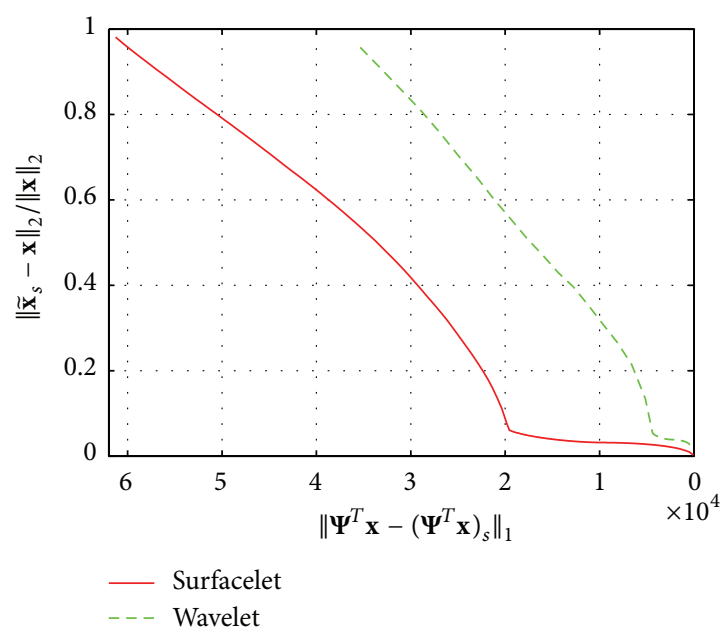

(b)

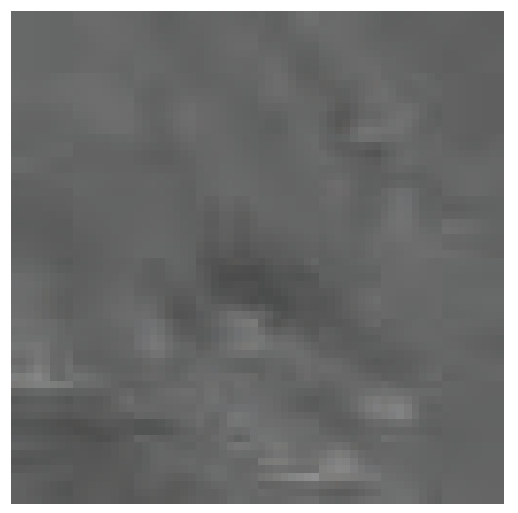

(d)

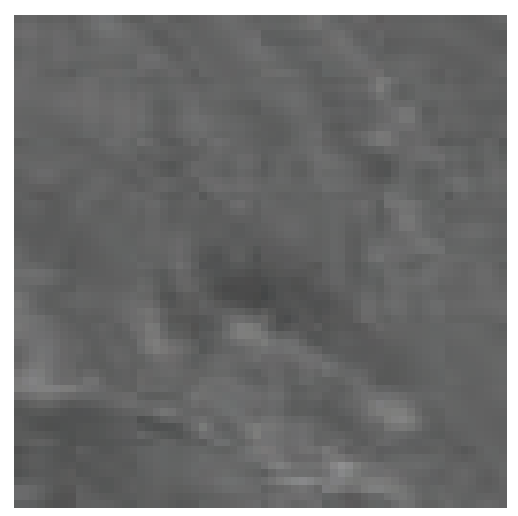

(e)

Figure 4: The $S$-term approximation errors and reconstructed images when $\left\|\Psi^{T} \mathbf{x}-\left(\Psi^{T} \mathbf{x}\right)_{S}\right\|_{1}=4 \times 10^{3}$, (a) Lunar Lake HSI data, (b) the $S$-term approximation error, (c) the original 5th band, (d) sparsely represented image using wavelet (PSNR $=33.12 \mathrm{~dB})$, and (e) sparsely represented image using surfacelet $(\mathrm{PSNR}=38.46 \mathrm{~dB}$ ).

arrays are different for different bands. The performance of both encoding schemes will be discussed in Section 3 .

In order to improve the performance of the recovered signal, a Gram-Schmidt orthogonalization (GSO) is used in CS encoding matrix. Given one band random encoding matrix $\overline{\boldsymbol{\Phi}}_{i}(1 \leq i \leq L)$ and the $n$th column denote as $\mathbf{v}_{n}$, the GSO is expressed by

$$
\begin{gathered}
\overline{\mathbf{v}}_{n}=\mathbf{v}_{n}-\sum_{m=1}^{n-1}\left\langle\mathbf{v}_{n}, \overline{\mathbf{v}}_{m}\right\rangle \mathbf{v}_{m}, \quad 2 \leq n \leq J, \\
\boldsymbol{\varphi}_{n}=\frac{\overline{\mathbf{v}}_{n}}{\left\|\overline{\mathbf{v}}_{n}\right\|_{2}^{2}}, \quad 1 \leq n \leq J .
\end{gathered}
$$

The new matrix $\Phi_{i}=\left(\varphi_{1}, \varphi_{2}, \ldots, \varphi_{J}\right)$ is adopted as the CS encoding matrix of one spectral band. The randomness of the matrix is not affected by GSO. In the following, columns of each $\Phi_{i}$ are processed with GSO.

2.3. Reconstruction Algorithm. For HSI $\mathbf{x}=\left(\mathbf{x}^{(1)}, \mathbf{x}^{(2)}, \ldots\right.$, $\left.\mathbf{x}^{(L)}\right) \in \mathbb{R}^{M \times N \times L}$, where $\mathbf{x}^{(i)}$ represents the $i$ th spectral band image, $M \times N$ represent the spatial dimensions, and $L$ represents the spectral depth of HSI. Let $\mathbf{x}_{i}=\operatorname{vec}\left(\mathbf{x}^{(i)}\right)(1 \leq$ $i \leq L)$, where $\mathbf{x}_{i}$ is a column vector of the $i$ th band HSI with size $M N \times 1$. The data acquisition model for CS is given by

$$
\mathbf{y}=\left[\begin{array}{c}
\mathbf{y}_{1} \\
\mathbf{y}_{2} \\
\vdots \\
\mathbf{y}_{L}
\end{array}\right]=\left[\begin{array}{cccc}
\boldsymbol{\Phi}_{1} & \mathbf{0}_{J \times M N} & \cdots & \mathbf{0}_{J \times M N} \\
\mathbf{0}_{J \times M N} & \boldsymbol{\Phi}_{2} & \cdots & \mathbf{0}_{J \times M N} \\
\vdots & & \ddots & \vdots \\
\mathbf{0}_{J \times M N} & \mathbf{0}_{J \times M N} & \cdots & \boldsymbol{\Phi}_{L}
\end{array}\right]\left[\begin{array}{c}
\mathbf{x}_{1} \\
\mathbf{x}_{2} \\
\vdots \\
\mathbf{x}_{L}
\end{array}\right],
$$

where $\Phi_{i} \in \mathbb{R}^{J \times M N}(J<M N)$ is a random encoding matrix for the $i$ th spectral band image and $\mathbf{y}_{i} \in \mathbb{R}^{J \times 1}$ is the acquired undersampled data. The sampling ratio is defined as

$$
\operatorname{CSR}=\frac{J}{M N}
$$

where CSR $<1$, which means the HSI are undersampled.

In this paper, ST is adopted to provide sparser representation of HSI data and is expected to reduce the reconstruction 


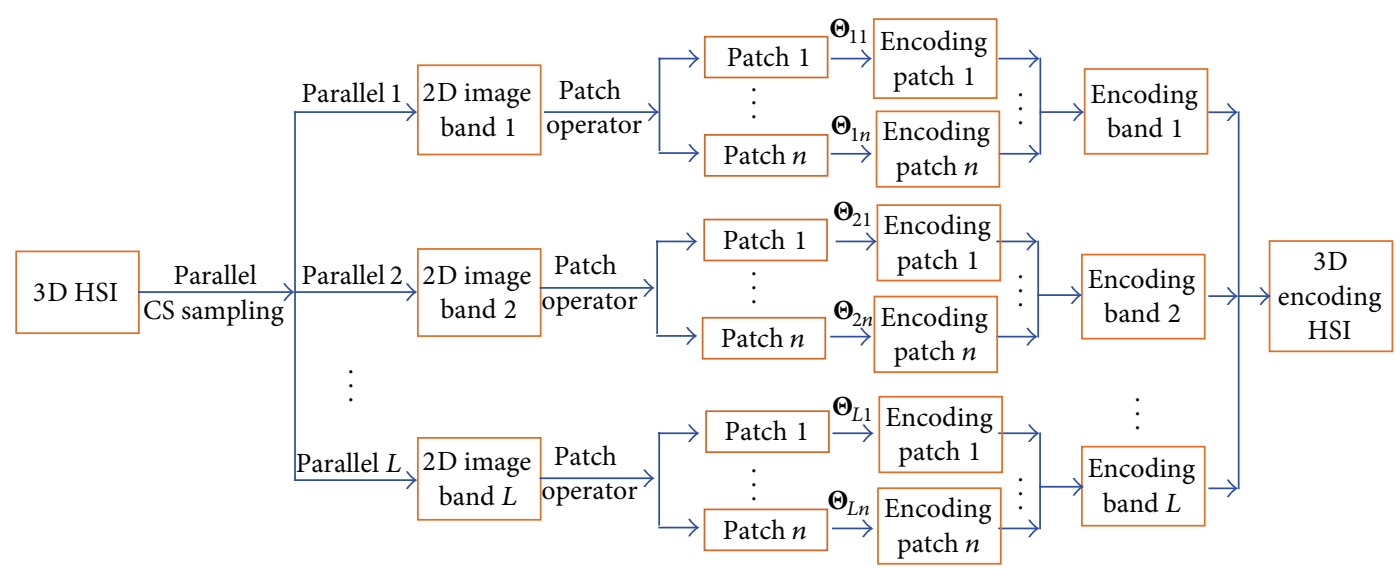

FIgURE 5: Parallel processing of compressive sampling on HIS.

error. Let $\Psi$ represent inverse ST and let $\Psi^{T}$ denote forward ST; CS recovers $\mathbf{x}$ by solving $[18,19]$

$$
\widehat{\boldsymbol{\alpha}}=\arg \min _{\boldsymbol{\alpha}}\left\{\lambda\|\boldsymbol{\alpha}\|_{1}+\|\mathbf{y}-\boldsymbol{\Phi} \Psi \boldsymbol{\alpha}\|_{2}^{2}\right\},
$$

where $\boldsymbol{\alpha}=\boldsymbol{\Psi}^{T} \mathbf{x},\|\cdot\|_{p}(p=1,2)$ stands for $l_{p}$-norm, and $\lambda$ is the regularization parameter which decides the tradeoff between the sparsity and the data fidelity. Many researchers seek for a simple and fast algorithm to solve (6), such as the conjugate gradient [30], Bregman iteration [31, 32], low rank reconstruction [33], and other methods. In this paper, we choose FISTA to solve (6) because of its simplicity and fast convergence, whose convergence rate is $\mathrm{O}\left(k^{-2}\right)$, where $k$ is an iteration counter [20].

To solve (6), the smallest Lipschitz constant [20] of the gradient $\|\mathbf{y}-\boldsymbol{\Phi} \Psi \boldsymbol{\alpha}\|_{2}^{2}$ is $G=2 \lambda_{\max }\left((\boldsymbol{\Phi} \Psi)^{T}(\boldsymbol{\Phi} \Psi)\right)$. The Lipschitz constant ensures the convergence of algorithm [34]. Taking $\boldsymbol{\alpha}_{0}=\mathbf{0}$ and $t_{1}=1$ as the initial values, the threshold is $\lambda / G$. For $k \geq 1$, solutions are found by iterating from (7) as follows:

$$
\begin{gathered}
\widehat{\boldsymbol{\alpha}}_{k}=\boldsymbol{\alpha}_{k}-\frac{1}{G}\left(\boldsymbol{\Psi}^{T} \boldsymbol{\Phi}^{T}\left(\boldsymbol{\Phi} \boldsymbol{\Psi} \boldsymbol{\alpha}_{k}-\mathbf{y}\right)\right), \\
\boldsymbol{\alpha}_{k}=\max \left\{\left|\widehat{\boldsymbol{\alpha}}_{k}\right|-\text { threshold, } 0\right\}_{+} \operatorname{sgn}\left(\widehat{\boldsymbol{\alpha}}_{k}\right), \\
t_{k+1}=\frac{1+\sqrt{1+4 t_{k}^{2}}}{2}, \\
\boldsymbol{\alpha}_{k+1}=\boldsymbol{\alpha}_{k}+\left(\frac{t_{k}-1}{t_{k+1}}\right)\left(\boldsymbol{\alpha}_{k}-\boldsymbol{\alpha}_{k-1}\right) .
\end{gathered}
$$

The maximum number of iterations of FISTA is set as 200 to achieve stable solutions. The final output is $\widehat{\mathbf{x}}=\boldsymbol{\Psi} \boldsymbol{\alpha}_{k}$.

2.4. Patch-Based Compressed Sensing with Surfacelet Reconstruction (PCSST). For a larger HSI data $\mathbf{x}$, bigger encoding matrix may exceed the memory of computer or leads long computation time. In this case, a patch-based sampling operation can be used. Let $\mathbf{R}$ be a patch operator and let
$\mathbf{R}_{l}(1 \leq l \leq L)$ divide the $l$ th band of $\mathbf{x}$ into $n$ patches, and then the data acquisition model for CS is given by

$$
\mathbf{y}=\left[\begin{array}{c}
\mathbf{y}_{1} \\
\mathbf{y}_{2} \\
\vdots \\
\mathbf{y}_{L}
\end{array}\right]=\left[\begin{array}{cccc}
\boldsymbol{\Theta}_{1} & \mathbf{0} & \cdots & \mathbf{0} \\
\mathbf{0} & \boldsymbol{\Theta}_{2} & \cdots & \mathbf{0} \\
\vdots & & \ddots & \vdots \\
\mathbf{0} & & \cdots & \Theta_{L}
\end{array}\right]\left[\begin{array}{c}
\mathbf{R}_{1} \mathbf{x}_{1} \\
\mathbf{R}_{2} \mathbf{x}_{2} \\
\vdots \\
\mathbf{R}_{L} \mathbf{x}_{L}
\end{array}\right]
$$

where $\mathbf{y}_{i}(1 \leq i \leq L)$ denotes measurements of patches in $i$ th band of $\mathbf{x}$ and $\Theta_{i}$ satisfies $\Theta_{i}=\left(\Theta_{i 1}, \Theta_{i 2}, \ldots, \Theta_{i n}\right)^{T}$, where $\boldsymbol{\Theta}_{i j}$ means random encoding on each block. In our scheme, the encoding on different patches of each band is different and these patches are nonoverlapped.

PCSST allows implementing the proposed method on a larger dataset. It has the potential to allow for parallel computing as shown in Figure 5 and also provides the possibility to reduce the complexity of sensor arrays since the sensing detectors independently sample the data in different patches.

\section{Experimental Results}

Experiments are conducted in three aspects. First, better reconstructed signal using ST than using wavelet is demonstrated. Second, improving the randomness of encoding matrix for each spectral band is shown to improve the recovery quality and the reconstruction performance of two encoding schemes is compared. Third, a patch-based CS encoding scheme is designed to deal with large data.

The HSI data is obtained from U.S. AVRIS website, including Moffet Field and Lunar Lake [35]. These data contain 224 spectral bands with spatial size $64 \times 64$, and every pixel is encoded with 16 bits. Linear interpolation is employed to fix the junk bands [36] and maintain the consistency of spectral lines, and all data are normalized.

To evaluate the performance, the mean-square-error (MSE), peak signal-to-noise ratio (PSNR), and structural 
similarity (SSIM) [37] are adopted as criteria to measure the reconstruction error. Their definitions are

$$
\begin{gathered}
\text { MSE }=\frac{1}{M \cdot N} \sum_{j=1}^{M} \sum_{i=1}^{N}(\widehat{x}(i, j)-x(i, j))^{2}, \\
\operatorname{PSNR}=10 \log _{10}\left(\frac{1}{\mathrm{MSE}}\right), \\
\operatorname{SSIM}(\hat{x}, x)=\frac{\left(2 \mu_{\hat{x}} \mu_{x}+C_{1}\right)\left(2 \sigma_{\widehat{x} x}+C_{2}\right)}{\left(\mu_{\hat{x}}^{2}+\mu_{x}^{2}+C_{1}\right)\left(\sigma_{\hat{x}}^{2}+\sigma_{x}^{2}+C_{2}\right)},
\end{gathered}
$$

where $x$ denotes the original image, $\mu_{x}$ and $\sigma_{x}$ are mean and standard deviation of $x, \widehat{x}$ stands for the recovered image, $\mu_{\widehat{x}}$ and $\sigma_{\widehat{x}}$ are mean and standard deviation of $\widehat{x} ; C_{1}, C_{2}$ are small constant, and $C_{1}=C_{2}=0.08$ is adopted in our work, which is used to avoid instability when either $\left(\mu_{\hat{x}}^{2}+\mu_{x}^{2}\right)$ or $\left(\sigma_{\hat{x}}^{2}+\sigma_{x}^{2}\right)$ is very close to zero [37]. Mean SSIM index is adopted in this paper. Simulations run on a dual core $2.5 \mathrm{GHz}$ CPU laptop with 4 GB RAM. A fast ST implementation in C language is adopted.

3.1. Surfacelet and Wavelet with 2D Encoding. To simulate the CS data acquisition, the HSI are undersampled by random encoding matrix $\Phi$. 50\% sampled data means $\Phi_{i} \in$ $\mathbb{R}^{\left(64^{2} / 2\right) \times 64^{2}}$ for $\mathbf{x}_{i} \in \mathbb{R}^{64^{2} \times 1}(1 \leq i \leq 224)$. In order to improve the quality of the recovered signal, Gram-Schmidt orthogonalization is used to obtain the CS encoding matrix. With the $2 \mathrm{D}$ encoding matrix (i.e., each band has the same encoding matrix), the ST-based reconstruction is compared with the 3D wavelets-based reconstruction. Daubechies filters " $\mathrm{db} 4$ " with 2 levels of decomposition is used, and there are 7 directional subbands for 3D wavelet. The multiscale pyramid with 2 decomposition levels is chosen, and there are 12 directional subbands for ST. The complexity of ST is $O\left(n \log _{10} n\right)$ and wavelet is $O(n)$.

In order to well discuss the relationship between $\lambda$ and PSNR, we compared the PSNR performance from $\lambda=10$ to $\lambda=10^{-4}$ as shown in Figure $6 . \lambda=10^{-2}$ is empirically chosen to give optimal PSNR in our work. As shown in Figure 7, ST significantly improves the PSNR of each spectral band more than $3 \mathrm{D}$ wavelet. Edges and curves are better reconstructed using ST than 3D wavelets as shown in Figure 8. At a given spatial location, the spectral line reconstructed using ST is much more consistent with the ground truth than wavelets as shown in Figure 9. Under different sampling ratios, ST achieves much better PSNRs than wavelet as shown in Figure 10, and more advantage of ST is seen at low ratios. Besides, ST shows better performance for the Lunar Lake dataset which has richer geometric textures than Moffet Field dataset. Running time (unit: second) based on ST and WT with different sampled rate is shown in Table 1.

3.2. $2 \mathrm{D}$ and $3 \mathrm{D}$ Encoding Matrix. The performance of $2 \mathrm{D}$ encoding and $3 \mathrm{D}$ encoding schemes is compared in this section. The recovered spatial images with two encoding schemes are shown in Figure 11. Edges and curves are better reconstructed using $3 \mathrm{D}$ encoding than $2 \mathrm{D}$ encoding. The

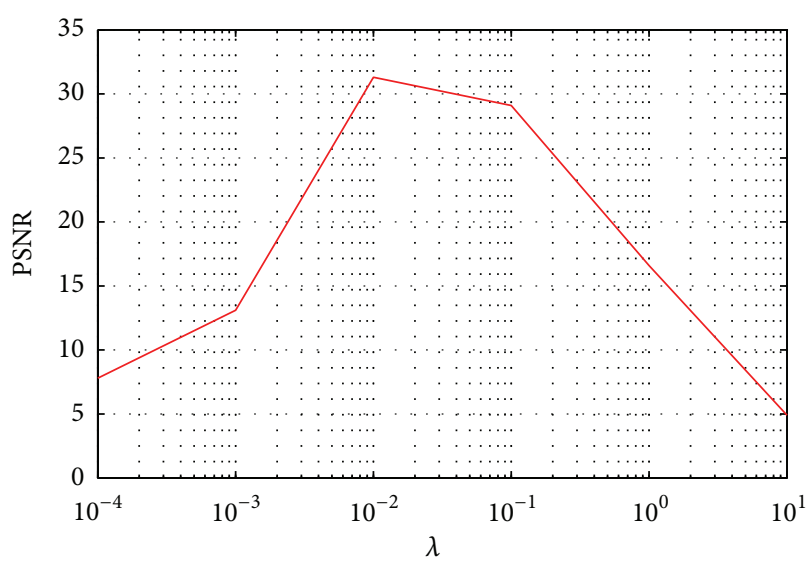

FIgURE 6: PSNR performance versus different $\lambda$. Note: test is performed on Lunar Lake dataset at sampling ratio $R=0.4$.

recovered spectral lines using $3 \mathrm{D}$ encoding are more consistent with ground truth than using $2 \mathrm{D}$ encoding as shown in Figure 12. The improvement is more obvious for Moffet Field dataset with textures in low intensities as shown in Figures 11(a) and 12(a). These results imply that increasing the encoding randomness among different bands will achieve better reconstruction.

3.3. Reconstruction with PCSST. We tested the methods on Lunar Lake data with size $256 \times 256 \times 224$. A reconstructed spectral band is shown in Figure 13. The image structures recovered by surfacelet are much sharper than wavelet.

\section{Conclusions}

Compressive sensing is a new sampling theorem. In this paper, the surfacelet transform is introduced into hyperspectral image reconstruction from compressive sampled data. The surfacelet transform is a directional multiresolution transform for 3D data, which is applied to sparsify the hyperspectral images for the first time. Simulations are conducted in three aspects. First, better reconstructed signal using surfacelet than using wavelet is demonstrated. Second, improving the randomness of encoding matrix for each spectral band is shown to improve the recovery quality. Third, a patch-based CS encoding scheme is designed to deal with large data. It provides the possibility to reduce the complexity of sensor arrays because sensing detectors in different patches independently sample data. Experiments demonstrate that reconstruction of spectral lines and spatial images is significantly improved using the proposed method than using conventional three-dimensional wavelets.

In the future, our work includes the following two aspects.

The first aspect is optimizing sparse representation of hyperspectral imaging, for example, combining adaptive sparse representation [38-40] and surfacelet transform. An adaptive dictionary may provide a sparser representation leading to a lower reconstruction error. Therefore, it is meaningful to try a low-complexity training method in the future. 


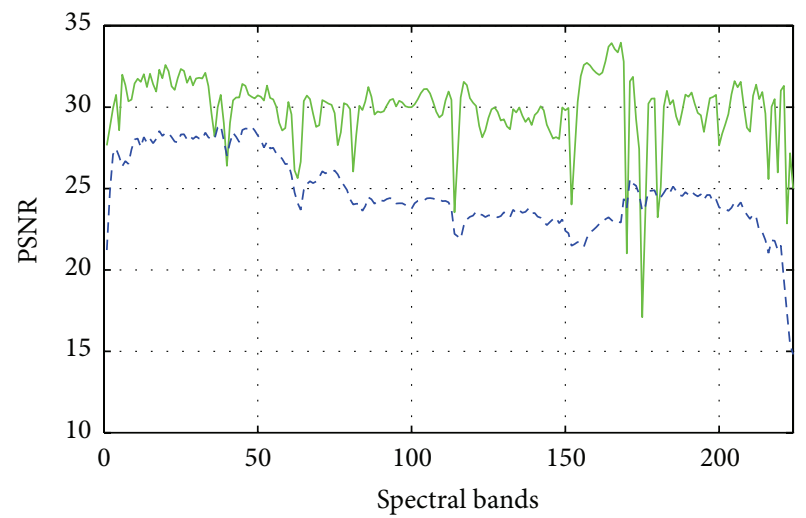

- Surfacelet

- . - Wavelet

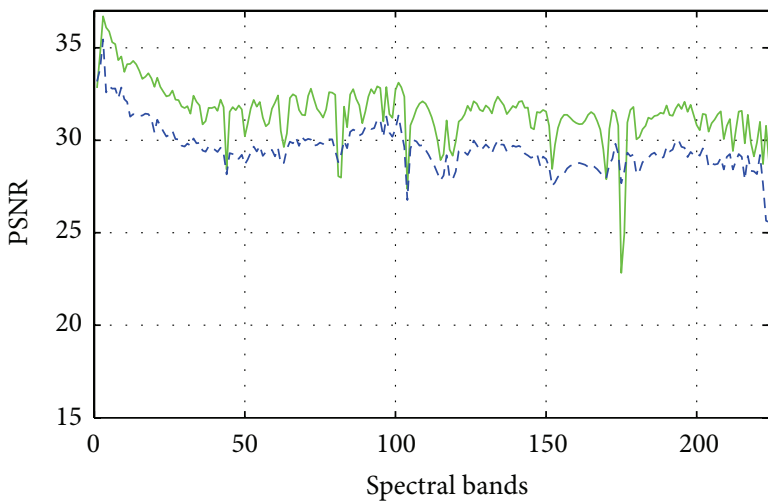

— Surfacelet

- _- Wavelet

(a)

(b)

FIGURE 7: PSNR performance using wavelet and surfacelet when sampling ratio is 0.40. (a) Moffet Field dataset and (b) Lunar Lake dataset.

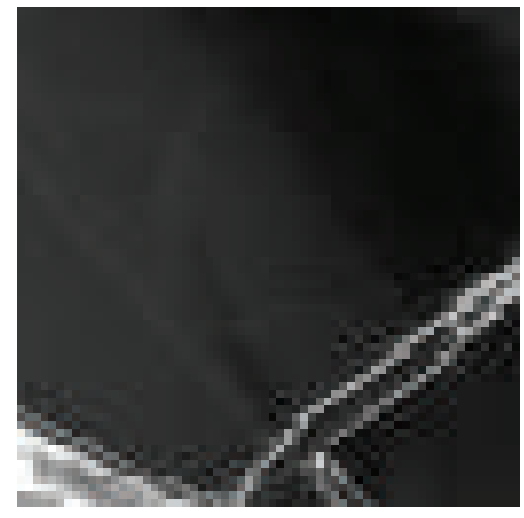

(a)

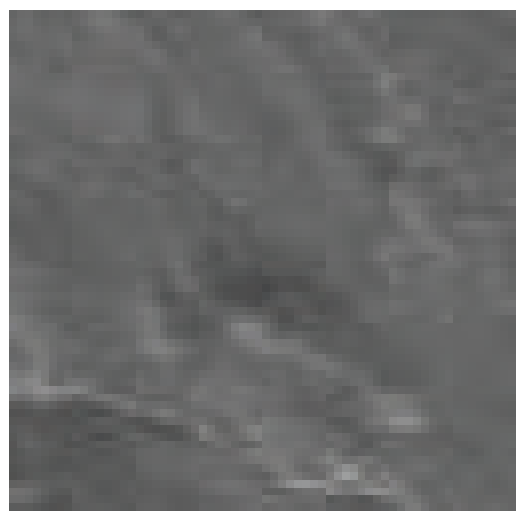

(d)

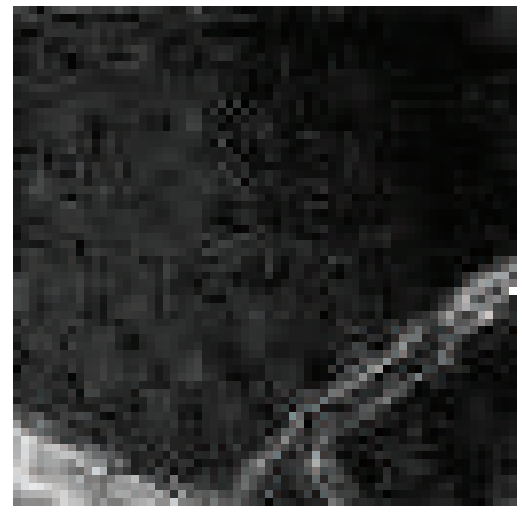

(b)

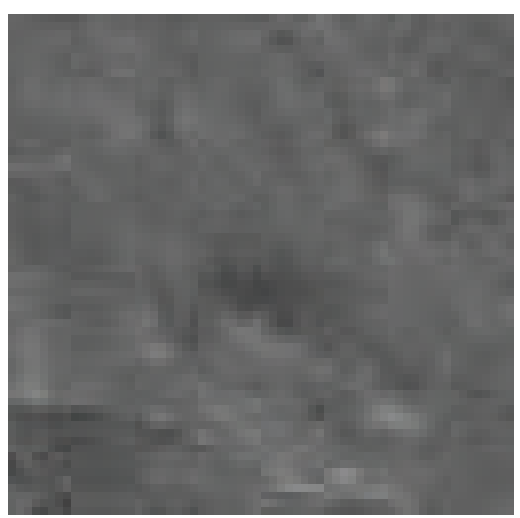

(e)

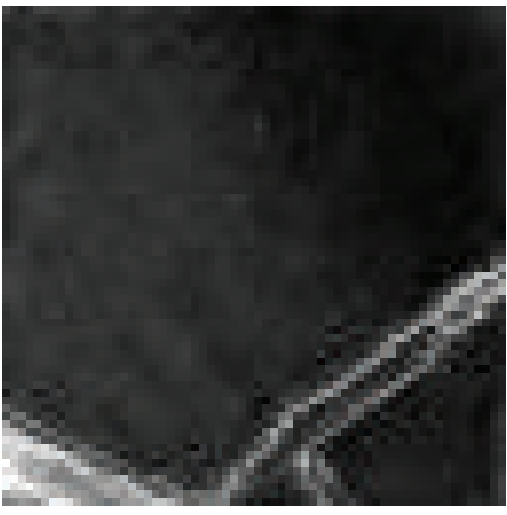

(c)

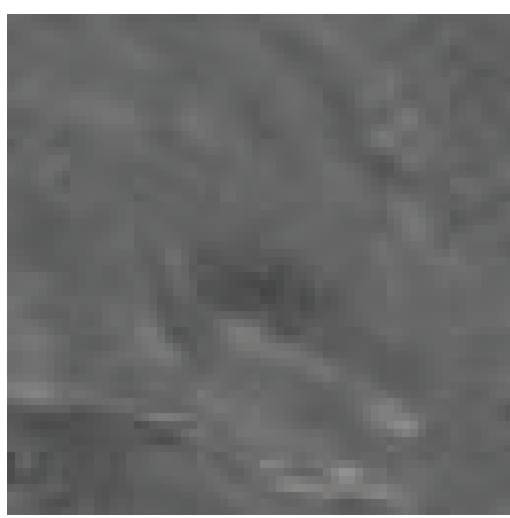

(f)

FIGURE 8: The reconstructed 199th band of Moffet Field and 5th band of Lunar Lake using wavelets or surfacelet when sampling ratio $R=0.40$. (a) and (d) are original Moffet Field and Lunar Lake bands, (b) PSNR $=24.31 \mathrm{~dB}$, SSIM $=0.9020$ and (e) PSNR $=32.91 \mathrm{~dB}$, SSIM $=0.9462$ are recovered bands using wavelet, and (c) PSNR $=30.75 \mathrm{~dB}$, SSIM $=0.9384$ and (f) PSNR $=35.88 \mathrm{~dB}$, SSIM $=0.9553$ are reconstructed bands using surfacelet. 


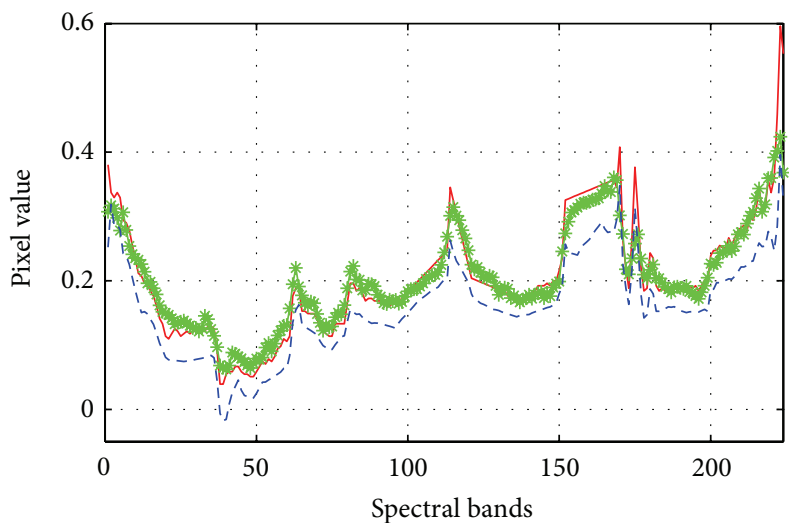

- Original spectral line

- - Surfacelet

- - Wavelet

(a)

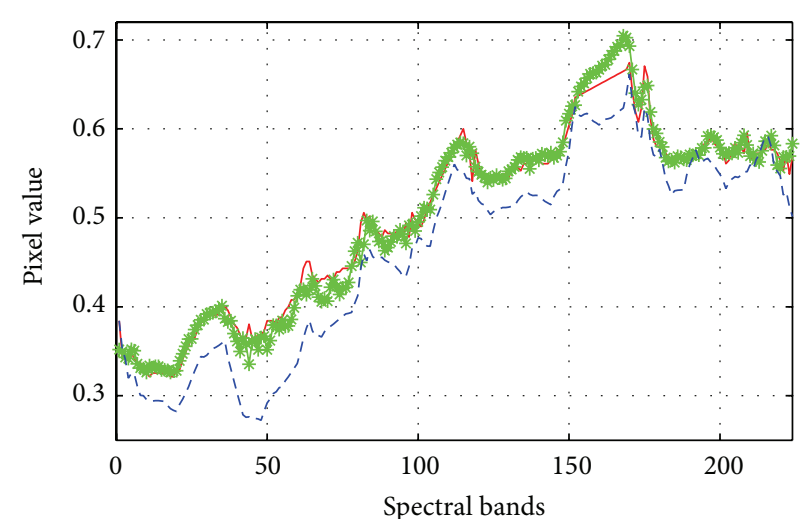

Original spectral line

*-Surfacelet

- . - Wavelet

FIgURE 9: Reconstructed spectral line when sampling ratio $R=0.40$. (a) Moffet Field dataset and (b) Lunar Lake dataset.

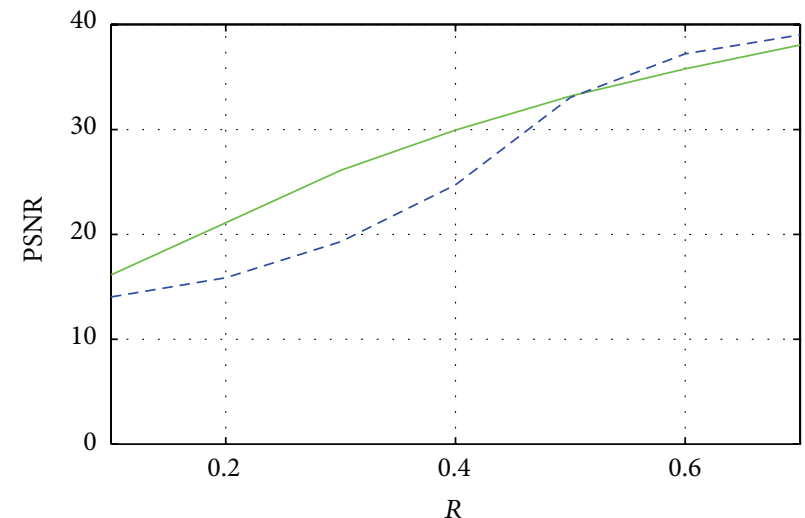

(a)

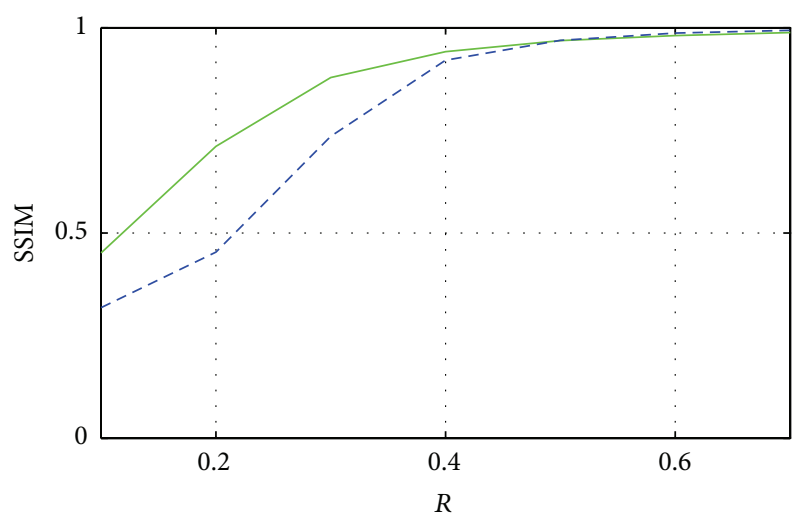

_ Surfacelet

- . - Wavelet

(c)

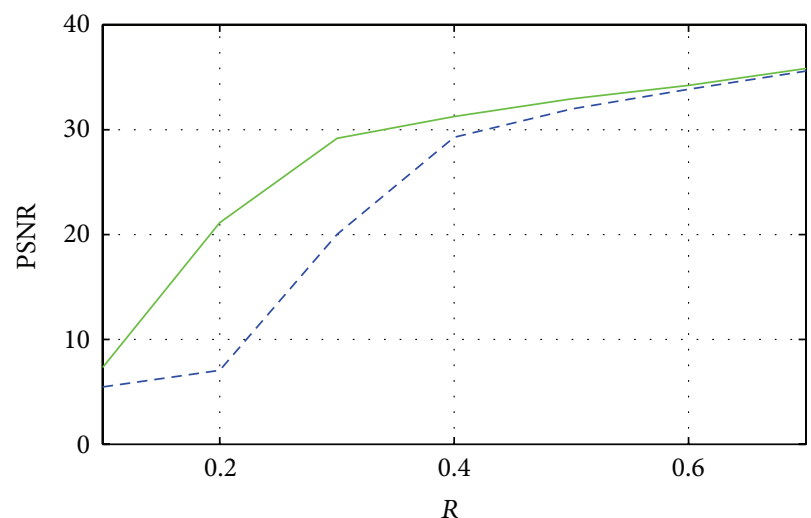

(b)

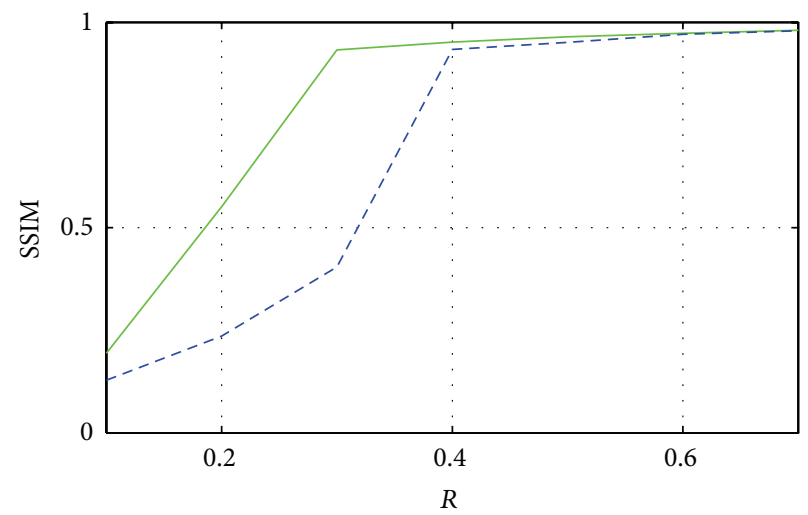

- Surfacelet

- - Wavelet

FIGURE 10: PSNR and SSIM at different sampling ratios. (a), (c) PSNR and SSIM for Moffet Field dataset and (b), (d) PSNR and SSIM for Lunar Lake dataset. 


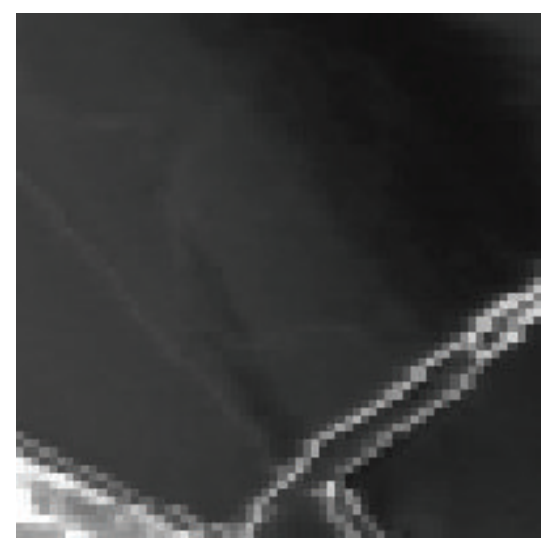

(a)

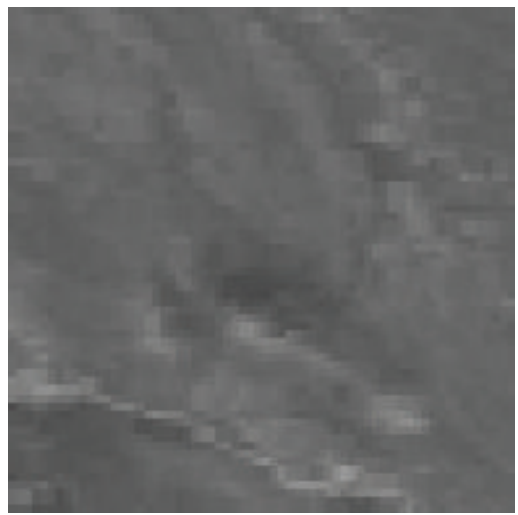

(d)

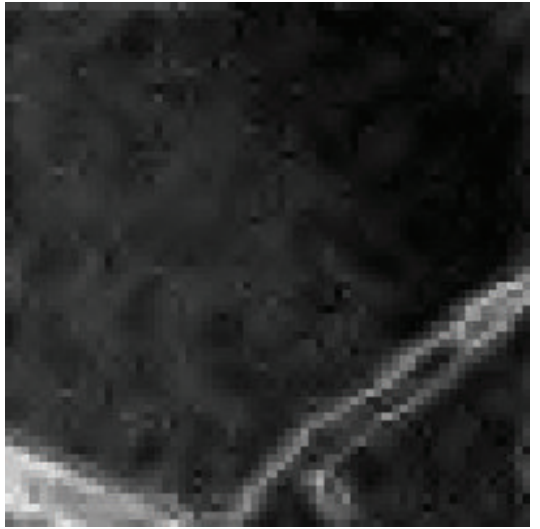

(b)

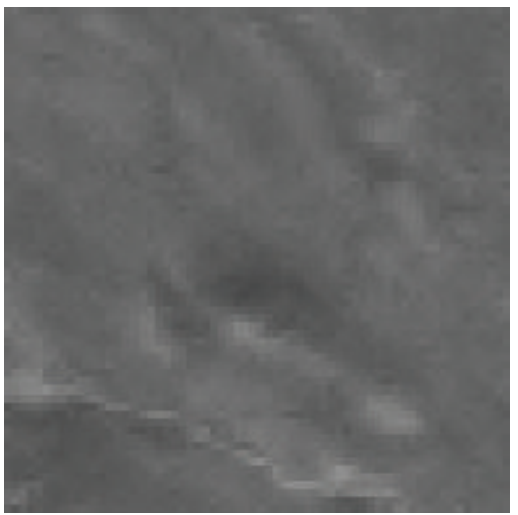

(e)

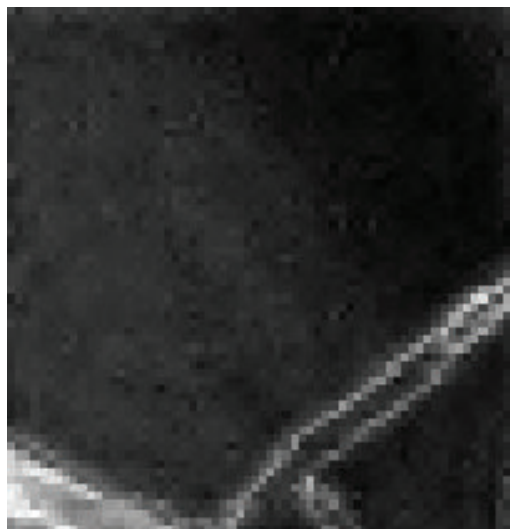

(c)

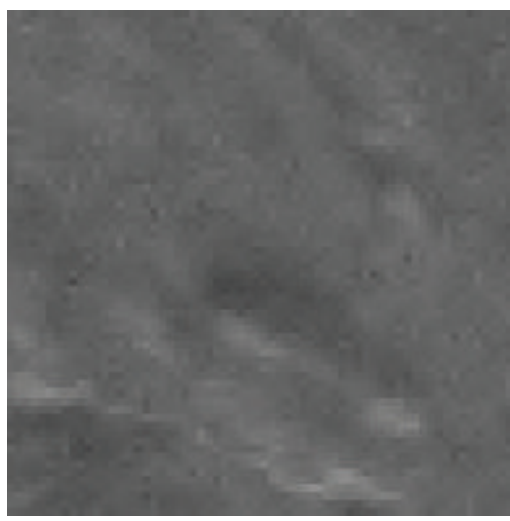

(f)

Figure 11: The reconstructed 199th band of Moffet Field and 5th band of Lunar Lake using 2D encoding and 3D encoding when sampling ratio $R=0.3$. (a) and (d) are original Moffet Field and Lunar Lake bands, (b) PSNR $=26.84 \mathrm{~dB}, \mathrm{SSIM}=0.9120$ and (e) PSNR $=33.65 \mathrm{~dB}$, SSIM $=0.9330$ are recovered bands using $2 \mathrm{D}$ encoding matrix for each band and (c) PSNR $=34.99 \mathrm{~dB}, \mathrm{SSIM}=0.9600$ and $(\mathrm{f})$ PSNR $=$ $37.84 \mathrm{~dB}, \mathrm{SSIM}=0.9701$ are reconstructed bands using different encoding matrix for each band.

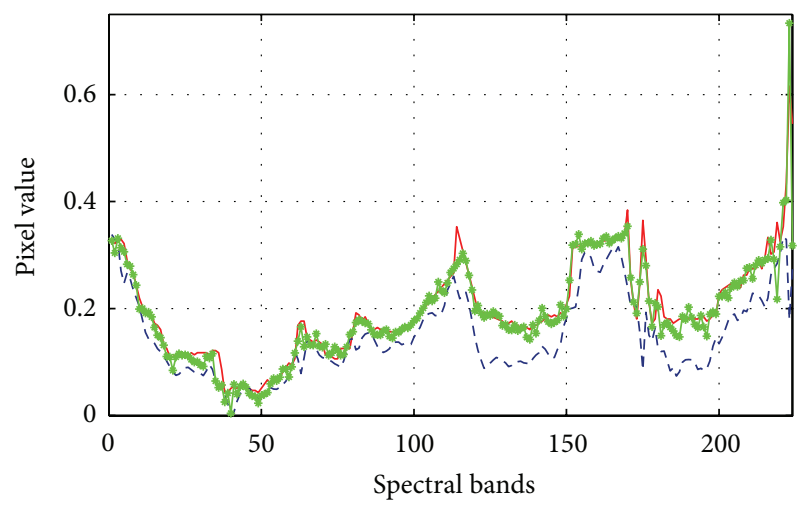

- Original spectral line

- - ST with $2 \mathrm{D}$ encoding

- ST with 3D encoding

(a)

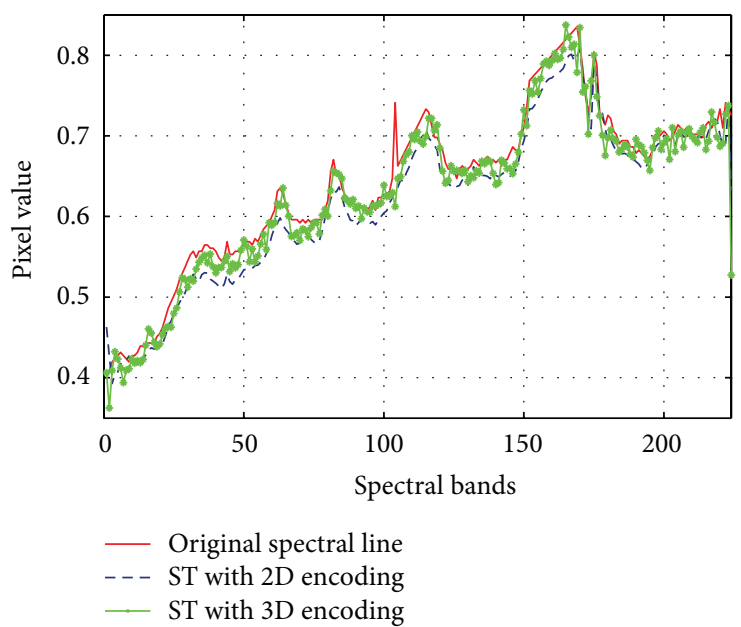

(b)

FIGURE 12: Reconstructed spectral line when sampling ratio $R=0.3$. (a) Moffet Field dataset and (b) Lunar Lake dataset. 
TABLE 1: Running time (unit: second) based on ST and WT with different sampled rate.

\begin{tabular}{lccccccrr}
\hline Sampling ratio & 0.1 & 0.2 & 0.3 & 0.4 & 0.5 & 0.6 & 0.7 & 0.8 \\
\hline ST & 458.9 & 629.7 & 833.8 & 1002.9 & 1224.2 & 1381.8 & 1544.9 & 1758.7 \\
WT & 989.8 & 1115.5 & 1359.7 & 1639.9 & 1871.6 & 2089.6 & 2324.1 & 2452.9 \\
\hline
\end{tabular}

Note: the ST is implemented in C and WT is implemented in MATLAB.

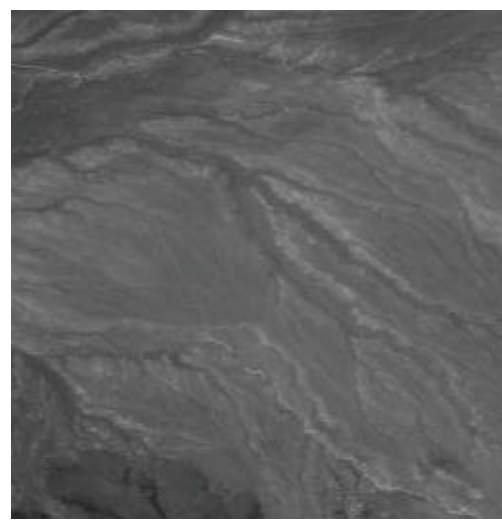

(a)

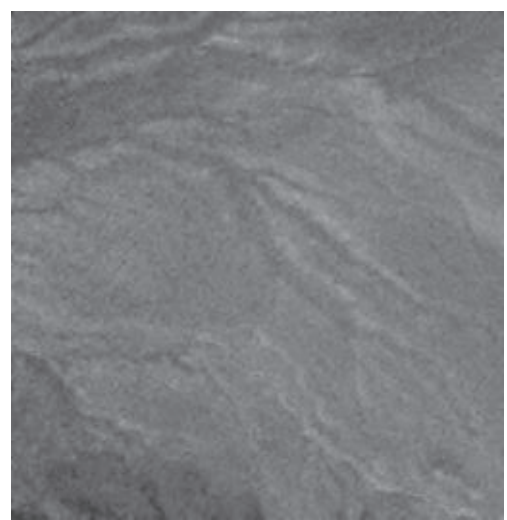

(b)

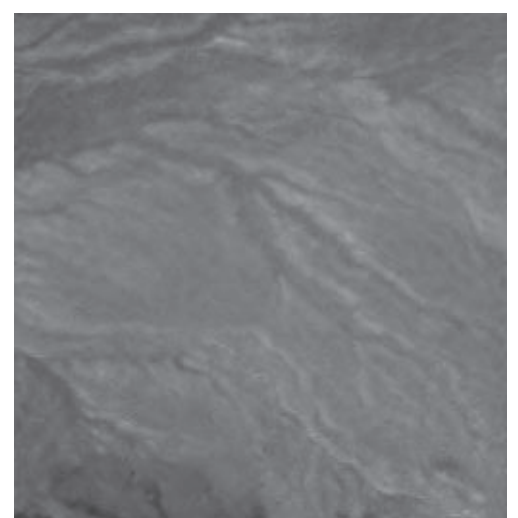

(c)

FIgURE 13: Patch-based compressed sensing ST reconstruction. (a) The original 5th band of $256 \times 256$ Lunar Lake dataset, (b) the patch-based reconstructed image using wavelet when undersampled rate $R=0.4$ and the PSNR $=32.92 \mathrm{~dB}, \mathrm{SSIM}=0.9551$, and $(\mathrm{c})$ the patch-based reconstructed image using ST when undersampled rate $R=0.4$ and the PSNR $=34.99 \mathrm{~dB}, \mathrm{SSIM}=0.9876$.

The second aspect is overlapping patches compressed sensing reconstruction. Overlapping patches can reduce the "block artifacts." But overlapping also introduces more encoded data and higher computations on image reconstructions. Trading the compressive sampling rate with image qualities using overlapping and speeding up the reconstruction algorithm [41] will be discussed in the future.

\section{Conflict of Interests}

The authors declare that there is no conflict of interests regarding the publication of this paper.

\section{Acknowledgments}

The authors wish to express their thanks to all reviewers for their comments. This work was supported in part by the National Natural Science Foundation of China (no. 61201045, no. 40971206, no. 61302174), Fundamental Research Funds for the Central Universities (no. 2013SH002), Open Fund from Key Lab of Digital Signal and Image Processing of Guangdong Province (no. 54600321, no. 2013GDDSIPL-07), Academic Innovation Team Building Project of Shantou University (no. ITC12002), and Scientific Research Foundation for the Introduction of Talent at Xiamen University of Technology (no. 90030606).

\section{References}

[1] A. F. H. Goetz, "Three decades of hyperspectral remote sensing of the Earth: a personal view," Remote Sensing of Environment, vol. 113, supplement 1, pp. S5-S16, 2009.
[2] J. Ma, "Single-Pixel remote sensing," IEEE Geoscience and Remote Sensing Letters, vol. 6, no. 2, pp. 199-203, 2009.

[3] Y. Zhao, J. Yang, and J. C. W. Chan, "Hyperspectral imagery super-resolution by spatial-spectral joint nonlocal similarity," IEEE Journal of Selected Topics in Applied Earth Observations and Remote Sensing, vol. 99, pp. 2671-2679, 2013.

[4] Y. Zhao, J. Yang, Q. Zhang, L. Song, Y. Cheng, and Q. Pan, "Hyperspectral imagery super-resolution by sparse representation and spectral regularization," EURASIP Journal on Advances in Signal Processing, vol. 2011, article 87, 2011.

[5] B. Aiazzi, L. Alparone, S. Baronti, C. Lastri, and M. Selva, "Spectral distortion in lossy compression of hyperspectral data," Journal of Electrical and Computer Engineering, vol. 2012, Article ID 850637, 8 pages, 2012.

[6] J. G. Kolo, S. A. Shanmugam, D. W. G. Lim, L.-M. Ang, and K. P. Seng, "An adaptive lossless data compression scheme for wireless sensor networks," Journal of Sensors, vol. 2012, Article ID 539638, 20 pages, 2012.

[7] Q. Du and J. E. Fowler, "Hyperspectral image compression using JPEG2000 and principal component analysis," IEEE Geoscience and Remote Sensing Letters, vol. 4, no. 2, pp. 201-205, 2007.

[8] C.-I. Chang, B. Ramakishna, J. Wang, and A. Plaza, "Exploitation-based hyperspectral image compression," Journal of Applied Remote Sensing, vol. 4, Article ID 041760, 2010.

[9] E. J. Candès, J. Romberg, and T. Tao, "Robust uncertainty principles: exact signal reconstruction from highly incomplete frequency information," IEEE Transactions on Information Theory, vol. 52, no. 2, pp. 489-509, 2006.

[10] D. L. Donoho, "Compressed sensing," IEEE Transactions on Information Theory, vol. 52, no. 4, pp. 1289-1306, 2006.

[11] X. Qu, Y. Hou, F. Lam, D. Guo, J. Zhong, and Z. Chen, "Magnetic resonance image reconstruction from undersampled measurements using a patch-based nonlocal operator," Medical Image Analysis, vol. 18, no. 6, pp. 843-856, 2014. 
[12] W. Hao, J. Li, X. Qu, and Z. Dong, "Fast iterative contourlet thresholding for compressed sensing MRI," Electronics Letters, vol. 49, no. 19, pp. 1206-1208, 2013.

[13] J. Xiong and Q. Tang, "1-bit compressive data gathering for wireless sensor networks," Journal of Sensors, vol. 2014, Article ID 805423, 8 pages, 2014.

[14] C. Caione, D. Brunelli, and L. Benini, "Compressive sensing optimization for signal ensembles in WSNs," IEEE Transactions on Industrial Informatics, vol. 10, no. 1, pp. 382-392, 2014.

[15] M. F. Duarte, M. A. Davenport, D. Takbar et al., "Single-pixel imaging via compressive sampling: building simpler, smaller, and less-expensive digital cameras," IEEE Signal Processing Magazine, vol. 25, no. 2, pp. 83-91, 2008.

[16] N. V. Aravind, K. Abhinandan, V. V. Acharya, and D. S. Sumam, "Comparison of OMP and SOMP in the reconstruction of compressively sensed hyperspectral images," in Proceedings of the International Conference on Communications and Signal Processing (ICCSP '11), pp. 188-192, Hamirpur, India, February 2011.

[17] J. E. Fowler, "Compressive-projection principal component analysis," IEEE Transactions on Image Processing, vol. 18, no. 10, pp. 2230-2242, 2009.

[18] E. J. Candès, "The restricted isometry property and its implications for compressed sensing," Comptes Rendus Mathematique, vol. 346, no. 9-10, pp. 589-592, 2008.

[19] E. J. Candès, Y. C. Eldar, D. Needell, and P. Randall, "Compressed sensing with coherent and redundant dictionaries," Applied and Computational Harmonic Analysis, vol. 31, no. 1, pp. 59-73, 2011.

[20] A. Beck and M. Teboulle, "A fast iterative shrinkage-thresholding algorithm for linear inverse problems," SIAM Journal on Imaging Sciences, vol. 2, no. 1, pp. 183-202, 2009.

[21] B. Rasti, J. R. Sveinsson, M. O. Ulfarsson, and J. A. Benediktsson, "Hyperspectral image denoising using 3D wavelets," in Proceedings of the 32nd IEEE International Geoscience and Remote Sensing Symposium (IGARSS '12), pp. 1349-1352, July 2012.

[22] X. Tang and W. A. Pearlman, "Three-dimensional waveletbased compression of hyperspectral images," Hyperspectral Data Compression, pp. 273-308, 2006.

[23] S. Prasad, W. Li, J. E. Fowler, and L. M. Bruce, "Information fusion in the redundant-wavelet-transform domain for noiserobust hyperspectral classification," IEEE Transactions on Geoscience and Remote Sensing, vol. 50, no. 9, pp. 3474-3486, 2012.

[24] A. Karami, M. Yazdi, and G. Mercier, "Compression of hyperspectral images using discerete wavelet transform and tucker decomposition," IEEE Journal of Selected Topics in Applied Earth Observations and Remote Sensing, vol. 5, no. 2, pp. 444-450, 2012.

[25] Y. M. Lu and M. N. Do, "Multidimensional directional filter banks and surfacelets," IEEE Transactions on Image Processing, vol. 16, no. 4, pp. 918-931, 2007.

[26] X. Qu, W. Zhang, D. Guo, C. Cai, S. Cai, and Z. Chen, "Iterative thresholding compressed sensing MRI based on contourlet transform," Inverse Problems in Science and Engineering, vol. 18, no. 6, pp. 737-758, 2010.

[27] P. Feng, Y. Pan, B. Wei, W. Jin, and D. Mi, "Enhancing retinal image by the contourlet transform," Pattern Recognition Letters, vol. 28 , no. 4, pp. 516-522, 2007.

[28] M. N. Do and M. Vetterli, "The contourlet transform: An efficient directional multiresolution image representation," IEEE Transactions on Image Processing, vol. 14, no. 12, pp. 2091-2106, 2005.
[29] E. J. Candès and D. L. Donoho, "New tight frames of curvelets and optimal representations of objects with piecewise $C^{2}$ singularities," Communications on Pure and Applied Mathematics, vol. 57, no. 2, pp. 219-266, 2004.

[30] M. Lustig, D. Donoho, and J. M. Pauly, "Sparse MRI: the application of compressed sensing for rapid MR imaging," Magnetic Resonance in Medicine, vol. 58, no. 6, pp. 1182-1195, 2007.

[31] W. Yin, S. Osher, D. Goldfarb, and J. Darbon, "Bregman iterative algorithms for $l_{1}$-minimization with applications to compressed sensing," SIAM Journal on Imaging Sciences, vol. 1, no. 1, pp. 143$168,2008$.

[32] J.-F. Cai, S. Osher, and Z. Shen, "Linearized Bregman iterations for compressed sensing," Mathematics of Computation, vol. 78, no. 267, pp. 1515-1536, 2009.

[33] Y. Q. Zhao and J. Yang, "Hyperspectral image denoising via sparse representation and low-rank constraint," IEEE Transactions on Geoscience and Remote Sensing, vol. 53, pp. 296-308, 2015.

[34] K. Goebel and W. A. Kirk, "A fixed point theorem for transformations whose iterates have uniform Lipschitz constant," Studia Mathematica, vol. 47, pp. 135-140, 1973.

[35] AVIRIS Homepage, JPL, NASA, December 2010, http://www .nasa.gov/.

[36] P. Zhong and R. Wang, "Multiple-spectral-band CRFs for denoising junk bands of hyperspectral imagery," IEEE Transactions on Geoscience and Remote Sensing, vol. 51, no. 4, pp. 22602275, 2013.

[37] Z. Wang, A. C. Bovik, H. R. Sheikh, and E. P. Simoncelli, "Image quality assessment: From error visibility to structural similarity," IEEE Transactions on Image Processing, vol. 13, no. 4, pp. 600-612, 2004.

[38] E. le Pennec and S. Mallat, "Sparse geometric image representations with bandelets," IEEE Transactions on Image Processing, vol. 14, no. 4, pp. 423-438, 2005.

[39] X. Qu, D. Guo, B. Ning et al., "Undersampled MRI reconstruction with patch-based directional wavelets," Magnetic Resonance Imaging, vol. 30, no. 7, pp. 964-977, 2012.

[40] M. Aharon, M. Elad, and A. Bruckstein, "K-SVD: an algorithm for designing overcomplete dictionaries for sparse representation," IEEE Transactions on Signal Processing, vol. 54, no. 11, pp. 4311-4322, 2006.

[41] J. Yang, Y. Yamaguchi, and W.-M. Boerner, "Numerical methods for solving the optimal problem of contrast enhancement," IEEE Transactions on Geoscience and Remote Sensing, vol. 38, no. 2, pp. 965-971, 2000. 

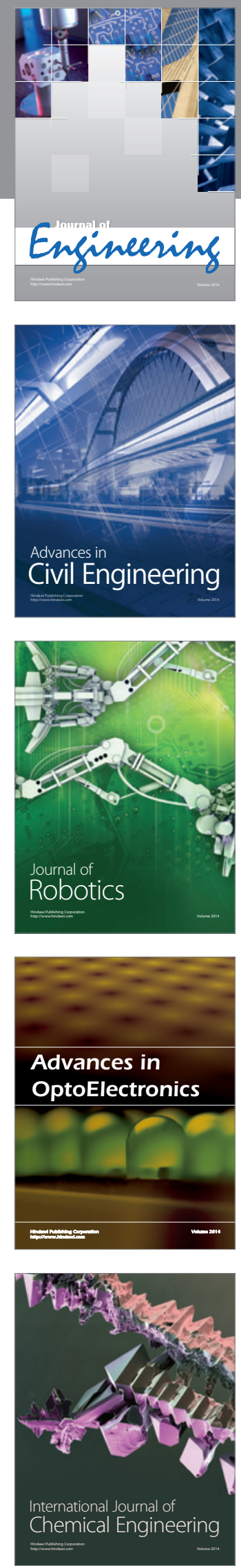

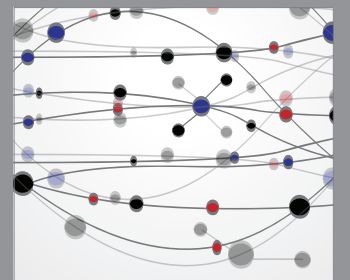

The Scientific World Journal
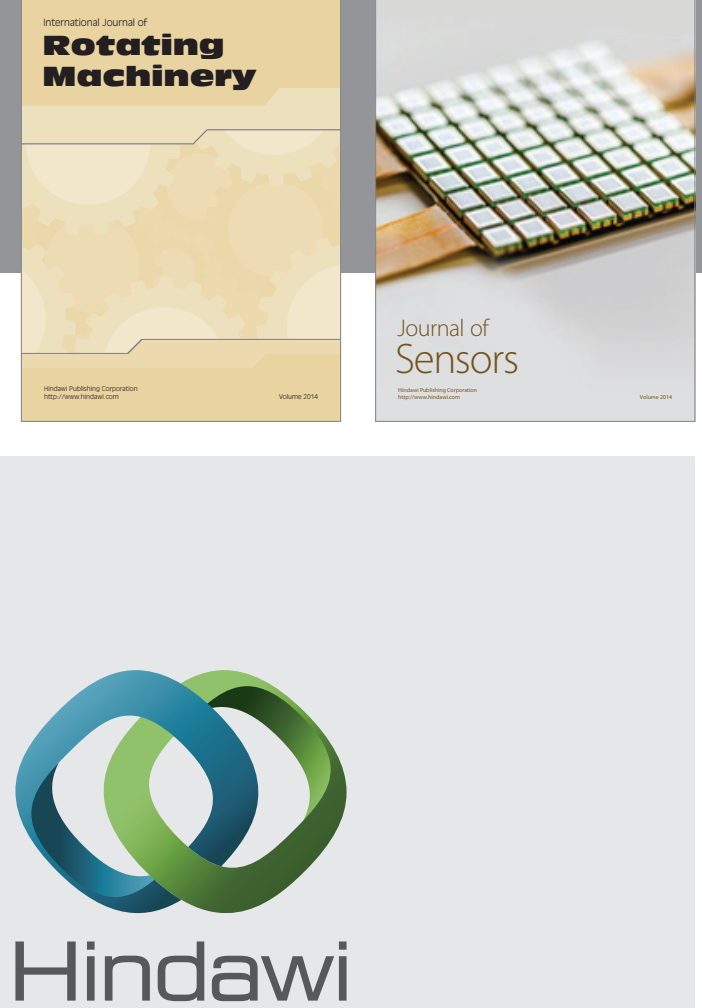

Submit your manuscripts at http://www.hindawi.com
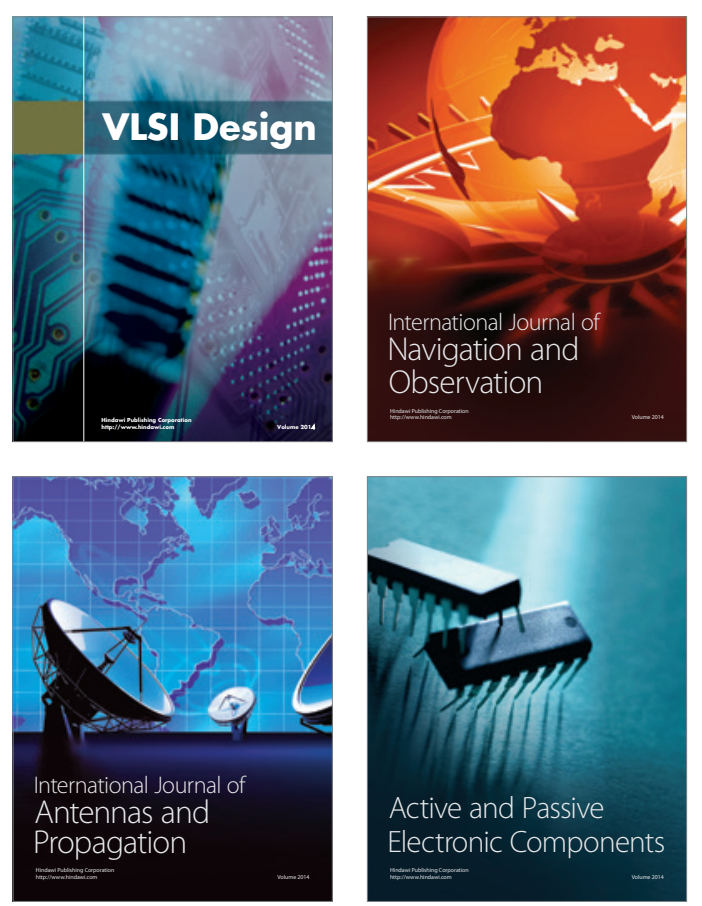
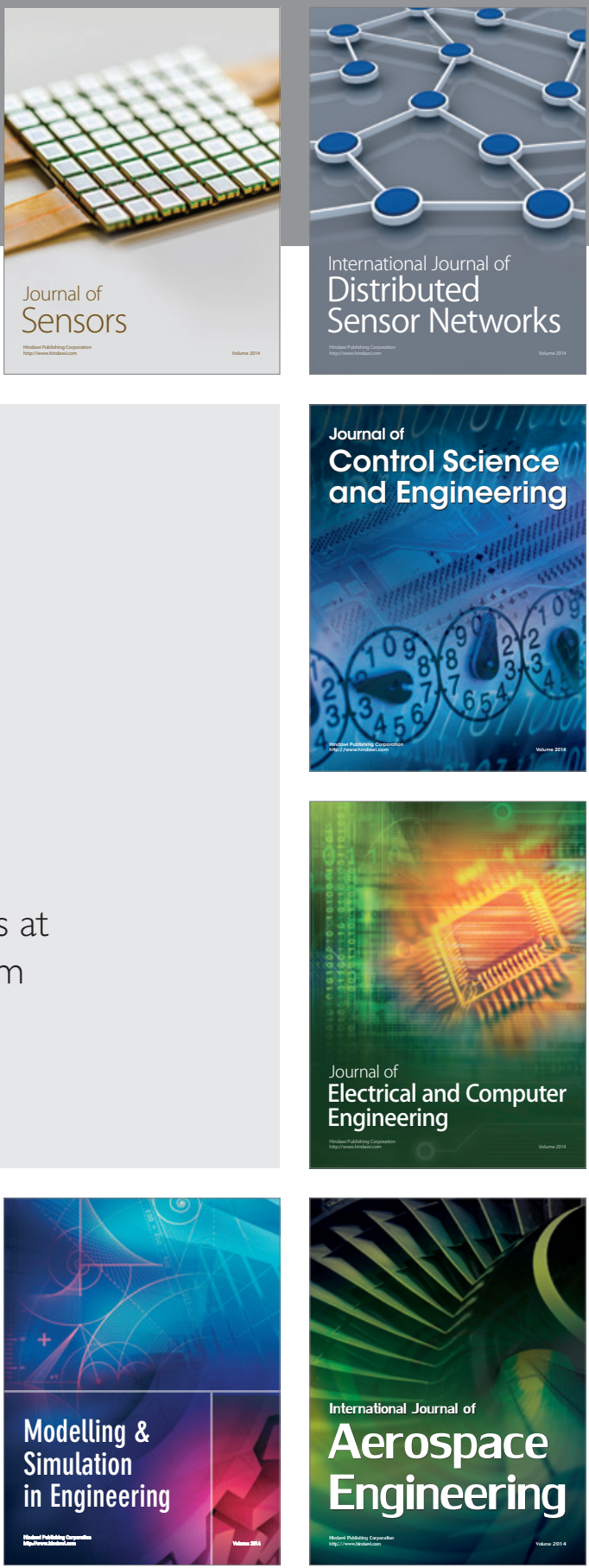

Journal of

Control Science

and Engineering
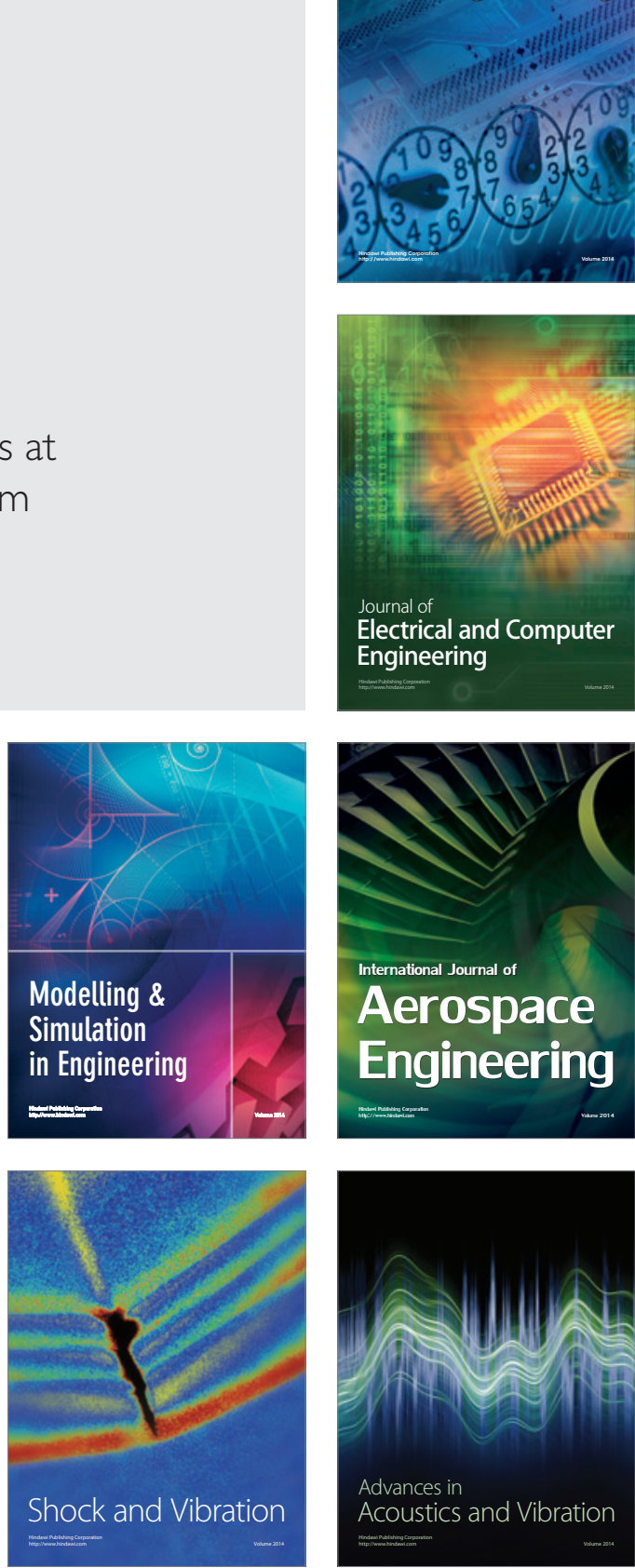